\title{
A Note on Integrating Factors of a Conformable Fractional Differential Equation
}

\author{
F. Martínez*, I. Martínez, S. Paredes \\ Departamento de Matemática Aplicada y Estadística, Universidad Politécnica de Cartagena, Cartagena, \\ España.
}

*Corresponding author. Tel.: +34968325586; email: f.martinez@upct.es

Manuscript submitted April 10, 2020; accepted June 28, 2020.

doi: 10.17706/ijapm.2020.10.4.143-152

\begin{abstract}
Recently exact fractional differential equations have been introduced, using the conformable fractional derivative. In this paper, we propose and prove some new results on the integrating factor. We introduce a conformable version of several classical special cases for which the integrating factor can be determined. Specifically, the cases we will consider are where there is an integrating factor that is a function of only $x$, or a function of only $y$, or a simple formula of $x$ and $y$. In addition, using the Conformable Euler's Theorem on homogeneous functions, an integration factor for the conformable homogeneous differential equations is established. Finally, the above results apply in some interesting examples.
\end{abstract}

Key words: A conformable Euler's theorem, conformable fractional derivative, exact fractional differential equation, integrating factor.

\section{Introduction}

For the many years, many definitions of fractional derivative have been introduced by various researchers. One of them is the Riemann-Liouville fractional derivative and the second one is the so-called Caputo derivative. These definitions are mostly used for mathematical models in many applications and are defined, respectively,

1) Riemann-Liouville definition. For $\alpha \in[n-1, n)$, the $\alpha$ derivative of $f$ is

$$
D_{t_{0}}^{\alpha}(f)(t)=\frac{1}{\Gamma(n-\alpha)} \frac{d^{n}}{d t^{n}} \int_{t_{0}}^{t} \frac{f(x)}{(t-x)^{\alpha-n+1}} d x
$$

2) Caputo definition. For $\alpha \in[n-1, n)$, the $\alpha$ derivative of $f$ is

$$
D_{t_{0}}^{\alpha}(f)(t)=\frac{1}{\Gamma(n-\alpha)} \int_{t_{0}}^{t} \frac{f^{(n)}(x)}{(t-x)^{\alpha-n+1}} d x
$$

Now, all definitions attempt to satisfy the usual properties of the standard derivative. The only property inherited by all definitions of fractional derivative is the linearity property. However, the following are the setbacks of one definition or another:

1) The Riemann-Liouville derivative does not satisfy $D_{a}^{\alpha}(1)=0 \quad\left(D_{a}^{\alpha}(1)=0\right.$ for the Caputo derivative.), if $\alpha$ is not natural number.

2) All fractional derivatives lost some of the basic properties that usual derivatives have such as the product rule, the quotient rule and the chain rule. 
3) All fractional derivatives do not satisfy: $D^{\alpha} D^{\beta} f=D^{\alpha+\beta} f$ in general.

4) The Caputo definition assumes that function $f$ is differentiable.

Recently, Khalil et al. introduced a new definition of fractional derivative called the conformable fractional derivative, [1]. Unlike other definitions, this new definition satisfies formulas of derivative of product and quotient of two functions and has a simpler chain rule. In addition, these authors introduce the conformable fractional derivative definition, the conformable integral definition, Rolle theorem and Mean value theorem for conformable fractional differentiable functions. In [2], Abdeljawad improves this new theory, establishing important elements of fractional calculus, such as: definitions of left and right conformable fractional derivatives and fractional integrals of higher order (i.e. of order $\alpha>1$ ), the fractional power series expansion, the fractional transform Laplace definition, fractional integration by parts formulas, chain rule and Gronwall inequality.

In the field of multivariate calculus, several works propose the extension of important concepts and results in the conformable sense. In [3], [4], the conformable partial derivative of the order $\alpha \in(0,1]$ of the real value of several variables and conformable gradient vector are defined; and a conformable version of Clairaut's Theorem for partial derivatives of conformable fractional orders is proven. In [5], two new results on homogeneous functions involving their conformable partial derivatives are introduced, specifically, homogeneity of the conformable partial derivatives of a homogeneous function and the conformable version of Euler's Theorem.

Finally, it is also a remarkable fact the large number of studies in the theory and application of fractional differential equations based on this new definition of derivative, which have been developed in a short time, [6]-[17].

The paper is organized as follows. In Section 2, the main concepts of conformable fractional calculus are presented. In Section 3, some results on exact fractional differential equations are recalled first, and then two new results on the integrating factor are proposed and proven. Specifically, the cases we will consider are where there is an integrating factor that is a function of only $x$, or a function of only $y$, or a simple formula of $x$ and $y$. Finally, an integrating factor is determined for homogeneous fractional differential equations.

\section{Basic Definitions and Tools}

Definition 2.1. [1]. Given a function $f:[0, \infty) \rightarrow R$. Then the conformable fractional derivative of $f$ of order $\alpha$, is defined by

$$
\left(T_{\alpha} f\right)(t)=\lim _{\epsilon \rightarrow 0} \frac{f\left(t+\varepsilon t^{1-\alpha}\right)-f(t)}{\varepsilon}
$$

For all $t>0,0<\alpha \leq 1$. If $f$ is $\alpha$-differentiable in some $(0, a), a>0$, and $\lim _{t \rightarrow 0^{+}}\left(T_{\alpha} f\right)(t)$ exist, then it is defined as

$$
\left(T_{\alpha} f\right)(0)=\lim _{t \rightarrow 0^{+}}\left(T_{\alpha} f\right)(t)
$$

As a consequence of the above definition, the following useful theorem is obtained, [1].

Theorem 2.1. If a function $f:[0, \infty) \rightarrow R$ is $\alpha$-differentiable at $t_{0}>0,0<\alpha \leq 1$, then $f$ is continuous at $t_{0}$.

It is easily shown that $T_{\alpha}$ satisfies the following properties, [1]

Theorem 2.2. Let $0<\alpha \leq 1$ and $f, g$ be $\alpha$-differentiable at a point $t>0$. Then

1) $T_{\alpha}(a f+b g)=a\left(T_{\alpha} f\right)+b\left(T_{\alpha} g\right), \forall a, b \in R$.

2) $T_{\alpha}\left(t^{p}\right)=p t^{p-\alpha}, \forall p \in R$.

3) $T_{\alpha}(\lambda)=0$, for all constant functions $f(t)=\lambda$. 


$$
\begin{aligned}
& \text { 4) } T_{\alpha}(f g)=f\left(T_{\alpha} g\right)+g\left(T_{\alpha} f\right) \text {. } \\
& \text { 5) } T_{\alpha}\left(\frac{f}{g}\right)=\frac{g\left(T_{\alpha} f\right)-f\left(T_{\alpha} g\right)}{g^{2}} \text {. } \\
& \text { 6) If, in addition, } f \text { is differentiable, then }\left(T_{\alpha} f\right)(t)=t^{1-\alpha} \frac{d f}{d t}(t) \text {. }
\end{aligned}
$$

The conformable fractional derivative of certain functions for above definition is given as follows:

1) $T_{\alpha}(1)=0$.

2) $T_{\alpha}(\sin (a t))=a t^{1-\alpha} \cos (a t), a \in R$.

3) $T_{\alpha}(\cos (a t))=-a t^{1-\alpha} \sin (a t), a \in R$.

4) $T_{\alpha}\left(e^{a t}\right)=a e^{a t}, a \in R$.

Further, many functions behave as in the usual derivative. Here are some formulas:

$$
\begin{aligned}
& \text { 1) } T_{\alpha}\left(\frac{1}{\alpha} t^{\alpha}\right)=1 \\
& \text { 2) } T_{\alpha}\left(e^{\frac{1}{\alpha} t^{\alpha}}\right)=e^{\frac{1}{\alpha} t^{\alpha}} \\
& \text { 3) } T_{\alpha}\left(\sin \left(\frac{1}{\alpha} t^{\alpha}\right)\right)=\cos \left(\frac{1}{\alpha} t^{\alpha}\right) \\
& \text { 4) } T_{\alpha}\left(\cos \left(\frac{1}{\alpha} t^{\alpha}\right)\right)=-\sin \left(\frac{1}{\alpha} t^{\alpha}\right)
\end{aligned}
$$

Remark 2.1. One should notice that a function could be $\alpha$-differentiable at a point but not differentiable. For example, take $f(t)=3 \sqrt[3]{t}$. Then $\left(T_{\frac{1}{3}} f\right)(0)=\lim _{t \rightarrow 0^{+}}\left(T_{\frac{1}{3}} f\right)(t)=1$, where $\left(T_{\frac{1}{3}} f\right)(t)=1$, for $t>0$. But $\frac{d f}{d t}(0)$ does not exist.

Theorem 2.3 (Mean Value Theorem). [6]. Let $a>0$ and $f:[a, b] \rightarrow R$ be a function that satisfies,

1) $f$ is continuous in $[a, b]$,

2) $f$ is $\alpha$-differentiable on $(a, b)$, for some $\alpha \in(0,1]$.

Then, there exists $c \in(a, b)$ such that

$$
\left(T_{\alpha} f\right)(c)=\left[\frac{f(b)-f(a)}{b-a}\right] c^{1-\alpha}
$$

Definition 2.2. [2]. The (left) conformable derivative starting from $a$ of a function $f:[a, \infty) \rightarrow R$ of $f$ of order $0<\alpha \leq 1$, is defined by

$$
\left(T_{\alpha}^{a} f\right)(t)=\lim _{\epsilon \rightarrow 0} \frac{f\left(t+\varepsilon(t-a)^{1-\alpha}\right)-f(t)}{\varepsilon}
$$

When $a=0$, it is written as $\left(T_{\alpha} f\right)(t)$. If $f$ is $\alpha$-differentiable in some $(a, b)$, then define

$$
\left(T_{\alpha}^{a} f\right)(a)=\lim _{t \rightarrow a^{+}}\left(T_{\alpha}^{a} f\right)(t)
$$

Note that if $f$ is differentiable, then $\left(T_{\alpha}^{a} f\right)(a)=(t-a)^{1-\alpha} \frac{d f}{d t}(t)$. Theorem 2.2 holds for Definition 2.2 when changing by $(t-a)$.

Theorem 2.4. (Chain Rule).[2]. Assume $f, g:(a, \infty) \rightarrow R$ be (left) $\alpha$-differentiable functions, where $0<\alpha \leq 1$. Let $h(t)=f(g(t))$. The $h(t)$ is $\alpha$-differentiable for all $t \neq a$ and $g(t) \neq 0$, therefore

$$
\left(T_{\alpha}^{a} h\right)(t)=\left(T_{\alpha}^{a} f\right)(g(t)) \cdot\left(T_{\alpha}^{a} g\right)(t) \cdot(g(t))^{\alpha-1}
$$

If $t=a$, then

$$
\left(T_{\alpha}^{a} h\right)(a)=\lim _{t \rightarrow a^{+}}\left(T_{\alpha}^{a} f\right)(g(t)) \cdot\left(T_{\alpha}^{a} g\right)(t) \cdot(g(t))^{\alpha-1}
$$

Now, there is the following definition for the $\alpha$-fractional integral of a function $f$ starting from $a \geq 0$.

Definition 2.3. [1]. $I_{\alpha}^{a}(f)(t)=\int_{a}^{t} \frac{f(x)}{x^{1-\alpha}} \cdot d x$, where the integral is the usual Riemann improper integral, and $\alpha \in(0,1]$. 
With the above definition, it was shown that

Theorem 2.5. [1]. $T_{\alpha}^{a} I_{\alpha}^{a}(f)(t)=f(t)$, for $t \geq a$, where $f$ is any continuous function in the domain of $I_{\alpha}$.

Lemma 2.1. [2]. Let $f:(a, b) \rightarrow R$ be differentiable and $\alpha \in(0,1]$. Then, for all $a>0$ we have,

$$
I_{\alpha}^{a} T_{\alpha}^{a}(f)(t)=f(t)-f(a)
$$

Finally, [3], [4], the conformable partial derivative of a real valued function with several variables is defined as follows:

Definition 2.4. Let $f$ be a real valued function with $n$ variables and $\boldsymbol{a}=\left(a_{1}, \ldots, a_{n}\right) \in R^{n}$ be a point whose $i t h$ component is positive. Then the limit

$$
\lim _{\epsilon \rightarrow 0} \frac{f\left(a_{1}, \ldots, a_{i}+\varepsilon a_{i}{ }^{1-\alpha}, \ldots, a_{n}\right)-f\left(a_{1}, \ldots, a_{n}\right)}{\varepsilon}
$$

If it exists, is denoted $\frac{\partial^{\alpha}}{\partial x_{i}^{\alpha}} f(\boldsymbol{a})$, and called the ith conformable partial derivative of $f$ of the order $\alpha \in$ $(0,1]$ at $a$.

Remark 2.2. If a real valued function $f$ with $n$ variables has all conformable partial derivatives of the order $\alpha \in(0,1]$ at $\boldsymbol{a}=\left(a_{1}, \ldots, a_{n}\right) \in R^{n}$, each $a_{i}>0$, then the conformable gradient of $f$ of the order $\alpha$ at $\mathbf{a}$ is

$$
\nabla^{\alpha} f(\boldsymbol{a})=\left(\frac{\partial^{\alpha}}{\partial x_{1}^{\alpha}} f(\boldsymbol{a}), \ldots, \frac{\partial^{\alpha}}{\partial x_{n}^{\alpha}} f(\boldsymbol{a})\right)
$$

Remark 2.3. Let $\alpha \in(0,1]$ and $f$ be a real valued function with $n$ variables defined on an open set $D \subset R^{n}$, such that, for all $\left(x_{1}, \ldots, x_{n}\right) \in D$, each $x_{i}>0$. Function $f$ is said to be in $C_{\alpha}(D, R)$ if all its conformable fractional partial derivatives of order $\alpha$ exists and are continuous on $D$, [5].

In [3], Clairaut's Theorem for conformable partial derivatives fractional orders presented as follows:

Theorem 2.6. Let $\alpha, \beta$ be positive constants such that $0<\alpha, \beta<1$. Assume That $f(x, y)$ is function for which $\frac{\partial^{\alpha}}{\partial x^{\alpha}}\left(\frac{\partial^{\beta} f(x, y)}{\partial y^{\beta}} f(x, y)\right)$ and $\frac{\partial^{\beta}}{\partial y^{\beta}}\left(\frac{\partial^{\alpha} f(x, y)}{\partial x^{\alpha}} f(x, y)\right)$ exists and are continuous over a domain $X \subset R^{n}$ such that for all $(x, y) \in X, \quad x, y>0$, then

For all $(x, y) \in X$.

$$
\frac{\partial^{\alpha}}{\partial x^{\alpha}}\left(\frac{\partial^{\beta} f(x, y)}{\partial y^{\beta}} f(x, y)\right)=\frac{\partial^{\beta}}{\partial y^{\beta}}\left(\frac{\partial^{\alpha} f(x, y)}{\partial x^{\alpha}} f(x, y)\right)
$$

\section{Conformable Fractional Differential Equation Reducible to Exact: Integrating Factor}

In this section, some results on exact fractional differential equations are recalled, [6].

Definition 3.1. Let $0<\alpha \leq 1$. A first order differential equation of the form $M(x, y) d x+N(x, y) d y=0$ is called $\alpha$-exact if there exists a function $\Phi(x, y)$ such that

$$
\frac{\partial^{\alpha} \Phi}{\partial y^{\alpha}}=M \text { and } \frac{\partial^{\alpha} \Phi}{\partial x^{\alpha}}=N
$$

Consequently,

$$
d^{\alpha} \Phi(x, y)=M(x, y) d x+N(x, y) d y=0
$$

From the properties of the conformable fractional derivative, we get $\Phi$ is a constant function.

Theorem 3.1. Let $0<\alpha \leq 1$. Let $M, N$ be a real valued function with two variables defined on a set $D$ and class $C_{\alpha}$ on $D$. Then $M(x, y) d x+N(x, y) d y=0$ is $\alpha$-exact if and only if

$$
\frac{\partial^{\alpha} N}{\partial x^{\alpha}}=\frac{\partial^{\alpha} M}{\partial y^{\alpha}}, \forall(x, y) \in D
$$


Definition 3.2. Let $0<\alpha \leq 1$. Let $M, N, \mu$ be a real valued function with two variables defined on a set $D$ and class $C_{\alpha}$ on $D$, with $x, y>0 \forall(x, y) \in D$. The function $\mu(x, y)$ is an integrating factor to the fractional differential equation $M(x, y) d x+N(x, y) d y=0$, if the fractional differential equation

$$
\mu(x, y) M(x, y) d x+\mu(x, y) N(x, y) d y=0
$$

is $\alpha$-exact.

Remark 3.1. To find an integrating factor $\mu(x, y)$, apply the $\alpha$-exactness condition on the equation (13)

$$
\frac{\partial^{\alpha}(\mu N)}{\partial x^{\alpha}}=\frac{\partial^{\alpha}(\mu M)}{\partial y^{\alpha}}
$$

That is,

$$
\mu\left(\frac{\partial^{\alpha} M}{\partial y^{\alpha}}-\frac{\partial^{\alpha} N}{\partial x^{\alpha}}\right)=N(x, y) \frac{\partial^{\alpha} \mu}{\partial x^{\alpha}}-M(x, y) \frac{\partial^{\alpha} \mu}{\partial y^{\alpha}}
$$

This is a fractional partial differential equation for the unknown function $\mu(x, y)$, which is more difficult to solve than the original fractional ordinary differential equation. However, for some special cases, equation (15) can be solved for an integrating factor.

\subsection{Special Cases}

\subsection{1. $\mu$ is a function of $x$ or $y$}

If $\mu$ is a function of $x$ only, that is, $\mu=\mu(x)$, [2], then

$$
\frac{\partial^{\alpha} \mu}{\partial x^{\alpha}}=T_{\alpha} \mu, \frac{\partial^{\alpha} \mu}{\partial y^{\alpha}}=0
$$

And equation (15) becomes

$$
N T_{\alpha} \mu=\mu\left(\frac{\partial^{\alpha} M}{\partial y^{\alpha}}-\frac{\partial^{\alpha} N}{\partial x^{\alpha}}\right)
$$

This implies that

$$
\frac{1}{\mu} T_{\alpha} \mu=\frac{1}{N}\left(\frac{\partial^{\alpha} M}{\partial y^{\alpha}}-\frac{\partial^{\alpha} N}{\partial x^{\alpha}}\right)
$$

Since $\mu(x)$ is a function of $x$ only, left hand side is a function of $x$ only. Hence, if an integrating factor of the form $\mu(x)$ is to exit, right hand side must be is a function of $x$ only. Then the integrating factor is, [6],

$$
\mu(x)=\exp \left[\int \frac{1}{N}\left(\frac{\partial^{\alpha} M}{\partial y^{\alpha}}-\frac{\partial^{\alpha} N}{\partial x^{\alpha}}\right) x^{\alpha-1} d x\right]
$$

Interchanging $M$ and $N$, and $x$ and $y$ in equation (15), one obtains an integrating factor for another special case, [6],

$$
\mu(y)=\exp [\int \underbrace{\frac{1}{M}\left(\frac{\partial^{\alpha} N}{\partial x^{\alpha}}-\frac{\partial^{\alpha} M}{\partial y^{\alpha}}\right)}_{\text {function of } y \text { only }} y^{\alpha-1} d y]
$$

Example 3.1. Consider

$$
2 x^{\alpha} y^{\alpha} d x+\left(2 x^{2 \alpha}-4 y^{2+\alpha}\right) d y=0
$$

For some $\alpha \in(0,1]$.

Solution. Here

$$
\frac{\partial^{\alpha}\left(2 x^{\alpha} y^{\alpha}\right)}{\partial y^{\alpha}}=2 \alpha x^{\alpha} \text { and } \frac{\partial^{\alpha}\left(2 x^{2 \alpha}-4 y^{2+\alpha}\right)}{\partial y^{\alpha}}=4 \alpha x^{\alpha}
$$


Thus the equation is not conformable $\alpha$-exact.

So,

$$
\frac{\partial^{\alpha}\left(2 x^{2 \alpha}-4 y^{2+\alpha}\right)}{\partial y^{\alpha}}-\frac{\partial^{\alpha}\left(2 x^{\alpha} y^{\alpha}\right)}{\partial y^{\alpha}}=2 \alpha x^{\alpha}
$$

Thus

$$
\frac{1}{\mu} T_{\alpha} \mu=\frac{2 \alpha x^{\alpha}}{2 x^{\alpha} y^{\alpha}}=\frac{\alpha}{y^{\alpha}}
$$

So now it is matter routine to solve the equation noticing that

$$
\mu(y)=\exp \left[\alpha \int \frac{1}{y^{\alpha}} y^{\alpha-1} d y\right]=y^{\alpha}
$$

\subsection{2. $\mu$ is a function of $x$ and $y$}

If $\mu$ is a function of $z$ only, where $z=z(x, y)$, [2], then

$$
\frac{\partial^{\alpha} \mu}{\partial x^{\alpha}}=\left(T_{\alpha} \mu\right)(z) \cdot z^{\alpha-1} \cdot \frac{\partial^{\alpha} z}{\partial x^{\alpha}}, \frac{\partial^{\alpha} \mu}{\partial y^{\alpha}}=\left(T_{\alpha} \mu\right)(z) \cdot z^{\alpha-1} \cdot \frac{\partial^{\alpha} z}{\partial y^{\alpha}}
$$

And equation (15) becomes

$$
\left(T_{\alpha} \mu\right)(z) \cdot z^{\alpha-1} \cdot\left(N \cdot \frac{\partial^{\alpha} z}{\partial x^{\alpha}}-M \cdot \frac{\partial^{\alpha} z}{\partial y^{\alpha}}\right)=\mu\left(\frac{\partial^{\alpha} M}{\partial y^{\alpha}}-\frac{\partial^{\alpha} N}{\partial x^{\alpha}}\right)
$$

This implies that

$$
\frac{1}{\mu(z)}\left(T_{\alpha} \mu\right)(z)=\frac{\frac{\partial^{\alpha} M}{\partial y^{\alpha}-\frac{\partial^{\alpha} N}{\partial x^{\alpha}}}}{z^{\alpha-1} \cdot\left(N \cdot \frac{\partial^{\alpha} z}{\partial x^{\alpha}}-M \cdot \frac{\partial^{\alpha} z}{\partial y^{\alpha}}\right)}
$$

Since $\mu(z)$ is a function of $z$ only, left hand side is a function of $z$ only. Hence, if an integrating factor of the form $\mu(z)$ is to exit, right hand side must be is a function of $z$ only. Then the integrating factor is,

$$
\mu(z)=\exp \left[\int \frac{\frac{\partial^{\alpha} M}{\partial y^{\alpha}-\frac{\partial^{\alpha} N}{\partial x^{\alpha}}}}{z^{\alpha-1} \cdot\left(N \cdot \frac{\partial^{\alpha} z}{\partial x^{\alpha}}-M \cdot \frac{\partial^{\alpha} z}{\partial y^{\alpha}}\right)} \cdot z^{\alpha-1} \cdot d z\right]
$$

Example 3.2. Find an integrating factor of the form $\mu(z)$, where $z=x y$, of the following equation

$$
\left(4 y^{1+\alpha}-6 x y^{\alpha}\right) d x+\left(6 x^{\alpha} y-4 x^{1+\alpha}\right) d y=0
$$

for Some $\alpha \in(0,1]$.

Solution. Here

$$
\frac{\partial^{\alpha}\left(4 y^{\alpha+1}-6 x y^{\alpha}\right)}{\partial y^{\alpha}}=4(1+\alpha) y-6 \alpha x
$$

And

$$
\frac{\partial^{\alpha}\left(6 x^{\alpha} y-4 x^{\alpha+1}\right)}{\partial x^{\alpha}}=6 \alpha y-4(1+\alpha) x
$$

Thus, the equation is not $\alpha$-exact.

Now, computing the conformable fractional partial derivative of function $\mu(z)$ with respect to $x$ and $y$, [3], then

$$
\frac{\partial^{\alpha} \mu}{\partial x^{\alpha}}=\left(T_{\alpha} \mu\right)(z) \cdot z^{\alpha-1} \cdot \frac{\partial^{\alpha} z}{\partial x^{\alpha}}=y^{\alpha} \cdot\left(T_{\alpha} \mu\right)(z)
$$


And

$$
\frac{\partial^{\alpha} \mu}{\partial y^{\alpha}}=\left(T_{\alpha} \mu\right)(z) \cdot z^{\alpha-1} \cdot \frac{\partial^{\alpha} z}{\partial y^{\alpha}}=x^{\alpha} \cdot\left(T_{\alpha} \mu\right)(z)
$$

Substituting these derivatives in equation (19) so that

$$
\frac{1}{\mu(z)}\left(T_{\alpha} \mu\right)(z)=\frac{(2-\alpha)}{z^{\alpha}}
$$

Finally, applying the fractional integral with respect to $z$ on both sides of above equation, an integrating factor is obtained

$$
\mu(x, y)=x^{2-\alpha} y^{2-\alpha}
$$

Example 3.3. Find an integrating factor of the form $\mu(z)$, where $z=x+y$, of the following equation

$$
\left(3 x^{\frac{1}{2}}-y^{\frac{1}{2}}\right) d x+\left(3 y^{\frac{1}{2}}+x^{\frac{1}{2}}\right) d y=0
$$

Solution. Here

$$
\frac{\partial^{\frac{1}{2}}\left(3 x^{\frac{1}{2}}-y^{\frac{1}{2}}\right)}{\partial y^{\frac{1}{2}}}=-\frac{1}{2} \text { and } \frac{\partial^{\frac{1}{2}}\left(3 y^{\frac{1}{2}}+x^{\frac{1}{2}}\right)}{\partial x^{\frac{1}{2}}}=\frac{1}{2}
$$

Now, computing the conformable fractional partial derivative of function $\mu(z)$ with respect to $x$ and $y$, [2], then

$$
\frac{\partial^{\frac{1}{2}} \mu}{\partial x^{\frac{1}{2}}}=\left(T_{\frac{1}{2}} \mu\right)(z) \cdot z^{-\frac{1}{2}} \cdot \frac{\partial^{\frac{1}{2}} z}{\partial x^{\frac{1}{2}}}=x^{\frac{1}{2}} \cdot z^{-\frac{1}{2}} \cdot\left(T_{\frac{1}{2}} \mu\right)(z)
$$

And

$$
\frac{\partial^{\frac{1}{2}} \mu}{\partial y^{\frac{1}{2}}}=\left(T_{\frac{1}{2}} \mu\right)(z) \cdot z^{-\frac{1}{2}} \cdot \frac{\partial^{\frac{1}{2}} Z}{\partial y^{\frac{1}{2}}}=y^{\frac{1}{2}} \cdot z^{-\frac{1}{2}} \cdot\left(T_{\frac{1}{2}} \mu\right)(z)
$$

Substituting these derivatives in equation (19) so that

$$
\frac{1}{\mu(z)}\left(T_{\frac{1}{2}} \mu\right)(z)=-\frac{1}{\sqrt{z}}
$$

Finally, applying the fractional integral with respect to $z$ on both sides of above equation, an integrating factor is obtained

$$
\mu(x, y)=\frac{1}{x+y}
$$

\subsubsection{Application to homogeneous fractional differential equation}

Let $\alpha \in(0,1]$ and $M, N$ are a real valued functions with two variables defined on an open set $D$ for witch $(t x, t y) \in D$ whenever $t>0$ and $(x, y) \in D$, with $x, y>0$, that satisfies

1) $M, N$ are homogeneous function of degree $r$

2) $M, N \in C_{\alpha}(D, R)$ Then an integrating factor of the homogeneous differential equation $M(x, y) d x+$ $N(x, y) d y=0$, is given by

$$
(x, y)=\frac{1}{x^{\alpha} \cdot M(x, y)+y^{\alpha \cdot N(x, y)}}
$$


providing $x^{\alpha} \cdot M(x, y)+y^{\alpha} \cdot N(x, y) \neq 0, \forall(x, y) \in D$

In effect, computing the conformable fractional partial derivatives of functions $\mu M$ and $\mu N$ with respect to $x$ and $y$, respectively, then

$$
\frac{\partial^{\alpha}}{\partial y^{\alpha}}\left(\frac{M(x, y)}{x^{\alpha} \cdot M(x, y)+y^{\alpha} \cdot N(x, y)}\right)=\frac{y^{\alpha} \cdot N(x, y) \cdot \frac{\partial^{\alpha} M(x, y)}{\partial y^{\alpha}}-\alpha M(x, y) N(x, y)-y^{\alpha} \cdot M(x, y) \cdot \frac{\partial^{\alpha} N(x, y)}{\partial y^{\alpha}}}{\left(x^{\alpha} \cdot M(x, y)+y^{\alpha} \cdot N(x, y)\right)^{2}}
$$

And

$$
\frac{\partial^{\alpha}}{\partial x^{\alpha}}\left(\frac{N(x, y)}{x^{\alpha} \cdot M(x, y)+y^{\alpha} \cdot N(x, y)}\right)=\frac{x^{\alpha} \cdot M(x, y) \cdot \frac{\partial^{\alpha} N(x, y)}{\partial x^{\alpha}}-\alpha M(x, y) N(x, y)-x^{\alpha} \cdot N(x, y) \cdot \frac{\partial^{\alpha} M(x, y)}{\partial x^{\alpha}}}{\left(x^{\alpha} \cdot M(x, y)+y^{\alpha} \cdot N(x, y)\right)^{2}}
$$

Finally, using the Conformable Euler's Theorem on homogeneous functions, [5], the result is followed.

Example 3.4. Consider

$$
\left(x^{2+\alpha}+y x^{1+\alpha}\right) d x+\left(2 x^{2+\alpha}+3 y^{2+\alpha}\right) d y=0
$$

For some $\alpha \in(0,1]$.

Since $M(x, y)=x^{2+\alpha}+y x^{1+\alpha}$ and $N(x, y)=2 x^{2+\alpha}+3 y^{2+\alpha}$ are homogeneous function of degree $2+\alpha$ and class $C_{\alpha}$ on open set $D$, with $x, y>0$, then above equation is an homogeneous differential equation and

$$
\mu(x, y)=\frac{1}{x^{\alpha}\left(x^{2+\alpha}+y x^{1+\alpha}\right)+y^{\alpha}\left(2 x^{2+\alpha}+3 y^{2+\alpha}\right)}=\frac{1}{x^{2+2 \alpha}+y x^{1+2 \alpha}+2 x^{2+\alpha} y^{\alpha}+3 y^{2+2 \alpha}}
$$

is an integrating factor of it.

\section{Conclusions}

The main objective of this work has been to generalize in the field of fractional calculus, some important results about the integrating factor for ordinary differential equations. The objective has been successfully achieved, so the definition of a partial derivative has been used to construct some results, such as: an integrating factor that is a function of only $x$, or a function of only $y$, an integrating factor that is a simple function of the variables $x$ and $y$, or an integrating factor for a differential equation homogeneous. It seems that the results obtained in this work correspond to the results obtained in the classic case.

\section{Conflict of Interest}

The authors declare no conflict of interest.

\section{Author Contributions}

F. Martínez and I. Martínez conceived the discussed idea and proved the results. S. Paredes wrote the paper and supervised the results of the work. All of authors had approved the final results and contributed to the final manuscript.

\section{References}

[1] Khalil, R., Al-Horani, M., Yousef, A., \& Sababheh, M. (2014). A new definition of fractional derivative. J. Comp. Appl. Math., 264(2014), 65-70.

[2] Abdeljawad, T. (2015). On conformable fractional calculus. J. Comp. Appl. Math., 57-66.

[3] Atangana, A., Baleanu, D., \& Alsaedi, A. (2018). New properties of conformable derivative. Open Math., 13, 57-63.

[4] Yazıcı-Gozütok, N., \& Gözütok, U. (2018). Multivariable conformable fractional calculus. Filomat, 32(1), 45-53. 
[5] Martínez, F., Martínez, I., \& Paredes, S. (2019). Conformable Euler's theorem on homogeneous functions. Comp. and Math. Methods, 1, e1048.

[6] Al-Horani, M., \& Khalil, R. (2018). Total fractional differential with applications to exact fractional differential equation. International Journal of Computer Mathematics, 95(6-7), 1444-1452.

[7] Iyiola, O. S., \& Nwaeze, E. R. (2016). Some new results on the new conformable fractional calculus with application using D’Alambert approach. Progr. Fract. Differ. Appl., 2(2), 1-7.

[8] Anderson, D. R., \& Ulness, D. J. (2015). Newly defined conformable derivatives. Advances in Dinamycal Systems and Applications, 10(2), 109-137.

[9] Al-Horani, M., Hammad, M. A., \& Khalil, R. (2016). Variations of parameters for local fractional nonhomogeneous linear-differential equations. J. Math. Computer Sci., 16, 147-153.

[10] Khalil, R., Al-Horani, M., \& Anderson D. (2016). Undetermined coefficients for local differential equations. J. Math. Computer Sci., 16, 140-146.

[11] Hammad, M. A., \& Khalil, R. (2014). Abel's formula and wronskian for conformable fractional differential equations. International Journal of Differential Equations and Applications, 13(2), 177-183.

[12] Hammad, M., \& Khalil, R. (2014). Legendre fractional differential equation and legendre fractional polynomials. Int. J. Appl. Math. Res., 3(3), 214-219.

[13] Ünal, E., \& Gökogak, A. (2017). Solution of conformable ordinary differential equations via differential transform method. Optik, 128, 264-273.

[14] Ünal, E., Gökogak, A., \& Cumhur, I. (2017). The operator method for local fractional linear differential equations. Optik, 131, 986-993.

[15] Bayour, B., \& Torres, D. (2017). Existence of solutions to a local fractional linear differential equation. J. Comp. Appl. Math., 312, 127-133.

[16] Silva, F. S., Moreira, D. M., \& Moret, M. A. (2018). Conformable Laplace transform of fractional differential equations. Axioms, 7.

[17] Masalmeh, M. (2017). Series Method to solve conformable fractional Riccati differential equation. Int. J. Appl. Math. Res., 6(1), 30-33.

Copyright (C) 2020 by the authors. This is an open access article distributed under the Creative Commons Attribution License which permits unrestricted use, distribution, and reproduction in any medium, provided the original work is properly cited ( $\underline{\text { CC BY } 4.0}$ ).

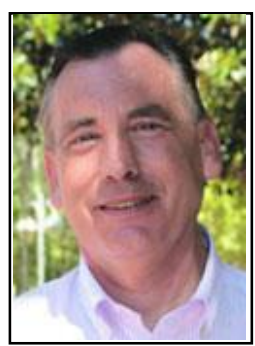

Francisco Martínez is a tenured associate professor at the Universidad Politécnica de Cartagena, Spain. He received PhD degree in physics from Universidad de Murcia in 1992. His research interests include the nonlinear dynamics methods and their applications, fractional calculus, fractional differential equations, multivariate calculus or special functions, and divulgation of mathematicsield of study should be lower-cased.

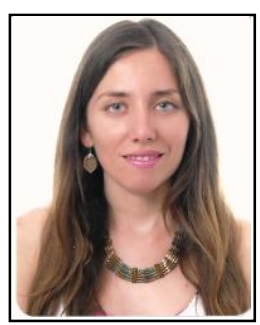

Inmaculada Martínez-Vidal is a PhD student of the industrial technologies program of the Universidad Politécnica de Cartagena, Cartagena, Spain. She is an industrial engineer from the Polytechnic University of Cartagena since 2012. She is doing her doctoral thesis in the field of fractional calculus and its applications to physics and engineering. She works as an engineer in the Spanish public company NAVANTIA, a benchmark in the design and construction of military ships and high-tech civilian ships and collaborates 
WITH the Departamento de Matemática Aplicada y Estadística of the Universidad Politécnica de Cartagena in the divulgation of mathematics.

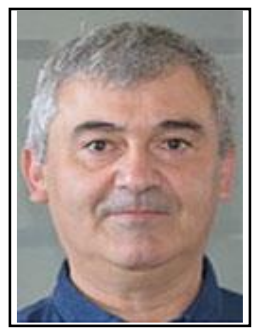

Silvestre Paredes is a tenured associate professor at the Universidad Politécnica de Cartagena, Spain. He received a PhD degree in mathematics from the Universidad de Valencia in 1995. His research interests include the study of large-scale structure of the universe, galaxies distribution, cosmology and astronomy, fractals and applications and fractional calculus. 